\title{
Sociocultural Settings Influence the Emergence of Prelinguistic Deictic Gestures
}

\author{
Dorothe Salomo \\ Max-Planck-Research Group Communication \\ before Language and Max-Planck-Institute \\ for Psycholinguistics
}

\author{
Ulf Liszkowski \\ Max-Planck-Research Group Communication \\ before Language, Max-Planck-Institute for \\ Psycholinguistics, and University of Hamburg
}

\begin{abstract}
Daily activities of forty-eight 8- to 15-month-olds and their interlocutors were observed to test for the presence and frequency of triadic joint actions and deictic gestures across three different cultures: Yucatec-Mayans (Mexico), Dutch (Netherlands), and Shanghai-Chinese (China). The amount of joint action and deictic gestures to which infants were exposed differed systematically across settings, allowing testing for the role of socialinteractional input in the ontogeny of prelinguistic gestures. Infants gestured more and at an earlier age depending on the amount of joint action and gestures infants were exposed to, revealing early prelinguistic sociocultural differences. The study shows that the emergence of basic prelinguistic gestures is socially mediated, suggesting that others' actions structure the ontogeny of human communication from early on.
\end{abstract}

Western-based research has identified infants' prelinguistic interaction and gestural communication skills as one of the most important milestones in early social and language development. First, early forms of triadic, object-related joint actions ("joint engagement"; Bakeman \& Adamson, 1984) help infants identify verbal reference to objects (Tomasello \& Todd, 1983). These triadic bouts of joint action are developmentally related to infants' word learning (Tomasello \& Farrar, 1986). Second, infants communicate with triadic, object-related gestures ("deictic gestures") in complex ways, including the comprehension and production of communicative, referential, and social intentions (for a recent overview, see Liszkowski, 2010). These deictic gestures are developmentally related to infants' acquisition of first words (Carpenter, Nagell, \& Tomasello, 1998; Harris, Barlow-Brown, \& Chasin, 1995) and to the emergence of syntactic word-word combinations (Iverson \& Goldin-Meadow, 2005).

We thank Lin Dongxian, Yi Salomo, Laura Shneidman, and Margret van Beuningen for their help with recruiting families. Thanks to Mireille Hassemer, Marloes van der Goot, Cornelia Seitz, and Merel Fokkema for data coding. We owe special thanks to the families in Mexico, the Netherlands, and China who offered us insights into their daily lives and allowed us to observe them at home.

Correspondence concerning this article should be addressed to Ulf Liszkowski, Max Planck Research Group Communication before Language, Max-Planck-Institute for Psycholinguistics, MPI for Psycholinguistics wundlaan 1, 6525 XD Nijmegen, Gelderland, The Netherlands. Electronic mail may be sent to ulf.liszkowski@mpi.nl.
However, still little is known about the very emergence of these prelinguistic interactions and gestures, especially from a sociocultural perspective. More cognitively oriented accounts emphasize that before sufficient cognitive skills are in place, socialinteractional input is not doing much work (although it will thereafter; Tomasello, 2008. Socialconstructivist accounts instead suggest that many behaviors are acquired socially through joint engagement and social scaffolding in the first place (Carpendale \& Lewis, 2004; Vygotsky, 1978). The central question is whether infants' gestures emerge independently of social interaction and only enable or constitute interaction and later verbal exchange, or whether infants' gestures emerge through socialinteractional experiences in the first place. Recent studies have established correlations between caregiver and infant gesture use (Liszkowski, Brown, Callaghan, Takada, \& De Vos, 2012; Liszkowski \& Tomasello, 2011; Rowe \& Goldin-Meadow, 2009), but the directionality has remained unclear. It is currently unknown whether caregivers gesture in response to infants' otherwise independently developing gestures or, alternatively, caregivers' interactions and gestures lead infants to gesture. In this respect, cultural comparisons are informative for development (Whiting, 1980). Humans do not

(C) 2012 The Authors

Child Development (c) 2012 Society for Research in Child Development, Inc. All rights reserved. 0009-3920/2012/8404-0014

DOI: $10.1111 /$ cdev.12026 
develop outside cultural contexts, and socialization is a pervasive feature of human development. If there were differences across different sociocultural settings in how interlocutors engage young infants in joint action and deictic gestural communication, this would provide the grounds for a natural experiment to test the effects of interactional 'input' on the early ontogeny of prelinguistic gestures.

Several studies indeed suggest large variation in the sociocultural settings within which children develop. Seminal comparative work measured 3- to 10-year-old children's socialization and interaction across different cultures, showing how specific socioculturally defined settings shape children's social development (Whiting \& Whiting, 1975). Further studies have shown that language socialization differs vastly across cultures, with some cultures apparently lacking infant-directed speech or believing that talking to preverbal infants is inappropriate (Pye, 1986; Schieffelin \& Ochs, 1986; for an overview, see Lieven \& Stoll, 2009). There are also substantial cultural differences in parental beliefs and practices on how to raise children, ranging from early promotion of development (see Weisner, 2009) to its rather passive acceptance (e.g., Lancy, 2010). Further, differences in socialization goals regarding the independence and interdependence of children's relations to their families (Kagitcibasi, 2005) have qualitative effects on how parents interact with their infants already in the first few months of life, with more proximal, tactile faceto-face interactions corresponding to interdependent socialization goals, and more distal, visual-verbal interactions corresponding to independent socialization goals (e.g., Keller, 2007; Keller, Otto, Lamm, Yovsi, \& Kärtner, 2008).

Most of these studies have documented differences in the sociocultural settings within which infants develop (for an overview, see Hewlett, 1996), how these differences in socialization practices are rooted in cultural belief systems (Harkness \& Super, 1996), or how they affect development once children fully participate in the respective socialization practices and are competent linguistic interactants (e.g., Feiring \& Lewis, 1981). However, it has remained unknown whether sociocultural differences already impact the early, prelinguistic emergence of object-directed deictic gestures. Given that young infants' social environments vary substantially across cultural settings, systematic comparisons could thus provide the quasi-experimental conditions to investigate whether different socialinteractional inputs affect the very emergence of prelinguistic gestures.
In the current study, we tested the hypothesis that social-interactional input as part of enculturation processes influences the emergence of prelinguistic gestures. We aimed at showing that socialization does not only impact children's development once they engage with language in the social practices of their cultures, but that enculturation impacts already the very emergence of prelinguistic gestures. To test this hypothesis, we first established systematic differences in input measures across three different sociocultural settings by quantifying the amount of deictic gestures directed at infants, and the amount of time others engaged infants in object-directed joint actions. As a test, we then compared infants' use of deictic gestures across these cultural groups. We measured infants' production of deictic gestures that emerge some time between 8 and 15 months of age (Carpenter et al., 1998), specifically index-finger pointing. Index-finger pointing is a milestone in the early bidirectional understanding of referential communication (as indicated by Western-based research; Liszkowski \& Tomasello, 2011), and it is used communicatively in infants across various sociocultural settings (Liszkowski et al., 2012). However, it is still unknown whether index-finger pointing emerges as a consequence of social-interactional input, or whether it emerges independently and only results in interactional responses (Carpendale \& Carpendale, 2010). We chose three specific cultural settings to test our hypothesis: Yucatec-speaking Mayans in Yucatán Mexico, Mandarin-speaking Chinese in Shanghai, and Dutch-speaking Netherlanders in Nijmegen.

We chose Netherlanders as a reference sample that is comparable to most of the findings in the developmental literature based on Western European cultural settings. Socialization practices in the Netherlands are child centered, with caregivers providing attention for the child, but within limits of caregivers' interest and availability, and with the goal that children also learn to play by themselves (Harkness, Super, \& van Tijen, 2000). The other two cultures were chosen because their socialization practices do not only differ from European cultures but also from one another. Other than in most Western cultural settings, Mayans' cultural belief is that young children develop and learn largely independently of caregivers' behavior toward them (Gaskins, 2000). Anthropological reports of Mayan societies indeed suggest that caregivers hardly engage with their infants verbally (Brown, 2000; Pye, 1992) or in joint object play (Gaskins, 1996). Thus, under the hypothesis that socialization influences prelinguistic interaction, we expected less 
infant gestures in the Mayan compared to the Dutch sample. In contrast, Shanghai Chinese infants are at the center of attention, especially since the implementation of the one-child policy (Goh \& Kuczynski, 2010; Ho, 1989). Parents and grandparents are anxious to ensure that their only child receives the best possible care (Fong, 2007; Goh, 2006), and the societal system is generally gauged toward ever earlier education and promotion of development and achievement. It has been documented that Chinese parents, compared to their U.S. counterparts, gesture relatively more for their 4-year-olds, possibly reflecting their heightened interest in teaching and instructing them (Goldin-Meadow \& Saltzman, 2000). Thus, here we expected a difference in the opposite direction, with more infant gestures than in the Dutch sample. Any other possible pattern of differences between the three groups, albeit difficult to interpret, would reject the proposed socialization hypothesis. The null hypothesis of finding no differences between groups would be amenable to several interpretations and, for example, support a more cognitively oriented account, suggesting that socialization processes are not effective before infants gesture and actively participate in social exchanges (e.g., Tomasello, 2008).

We made natural observations of infants' daily activities. Natural observations are ecologically more valid than observations in predefined contexts, like laboratory-based experiments or elicitation tasks, in that they are more sensitive to capturing natural group differences. We conducted online scan samplings on different times of a day to determine how much of their time infants were naturally engaged in joint actions by any interactant (e.g., parents, relatives, adults, siblings, peers). Scan sampling is an established paper-and-pencil method in ethology and cultural anthropology, which is minimally intrusive and involves registering online the occurrence of predefined categories of behavior in a given time frame. In addition, we made video recordings on different times of a day to analyze in detail the forms and frequencies of infants' and their interlocutors' gestural communication.

Under the assumption that socialization affects the emergence of prelinguistic communication, we expected the Yucatec Mayans and Shanghai Chinese on the opposite extremes, with the Dutch covering some middle ground. In a first set of analyses we tested whether our three samples indeed differed from one another in the input they provided to their infants. We tested whether (a) Yucatec Mayans engaged their infants in less triadic interac- tions and used less deictic gestures for their infants compared to the Dutch, and (b) Shanghai Chinese engaged their infants more often in triadic interaction and used more deictic gestures for their infants compared to the Dutch. In a second set of analyses we looked at infants' deictic gestures as an outcome measure. We tested whether the predicted cultural group differences would pertain to infants' gesture use and, in particular, to their index-finger pointing. Further, we tested for positive correlations between the social-interactional input measures and infants' gestures. Importantly, differences on the cultural group level would be informative for the directionality of such correlations, as there was no a priori basis for differences between the agematched infants across the three cultural groups that would follow our predicted pattern.

We coded also other, more primary child-care activities, which we expected to be similar across cultural groups. Further, we coded for the proximity of caregivers as another factor that could possibly influence interaction patterns (e.g., Feiring \& Lewis, 1981). We chose an age interval that ranged from a period before to after the emergence of joint attentional behaviors and deictic gestures (as identified by Western-based research) to investigate the possibility of different developmental time courses across the cultural settings.

\section{Method}

\section{Participants}

Sixteen Yucatec Mayan infants (7 girls; mean age $=11$ months 29 days, range $=8.01-15.25), 16$ Dutch infants ( 9 girls; mean age $=11$ months 23 days, range $=8.04-15.03)$, and 16 Chinese infants (9 girls; mean age $=11$ months 26 days, range = 8.09-15.15) and their families and temporary interlocutors participated in the study. Infants were matched for age across all three groups following the initial data collection in Mexico. In each setting, half of the sample was between 8 and 11 months of age (the younger group) and half were between 12 and 15 months of age (the older group).

Yucatec Mayan infants were recruited in two small neighboring villages near the town Chemax on the Yucatán Peninsula (Mexico) with the help of a native assistant and several local people. The observer (first author) lived for about 6 weeks in a family in one of the focal villages. All families with infants in the appropriate age range were invited to participate in the study, and most families agreed. Infants in the Netherlands were recruited in Nijme- 
gen, a medium-sized Dutch city, by the same observer who had lived there for half a year. Parents had previously registered in a database to participate in infant studies and were contacted by phone. In China, infants were recruited in the metropolis Shanghai. The same observer spent about 5 weeks in Shanghai living with a local relative who assisted her in the recruitment process. Chinese families with infants in the appropriate age range were approached in various city quarters by two local Mandarin-speaking assistants and asked to participate. The families in all three cultures received a monetary gift for participation as well as a DVD with the video recordings of their session.

\section{Sample Characteristics}

The Yucatec Mayan families lived in traditional villages, remote from tourism centers and other cities, with no public transport. Mayan infants typically grow up in large families. All infants had between 6 and 11 siblings - apart from one infant who was a firstborn. Older siblings often took care of their younger brothers and sisters. Almost all the men in the two participating villages were subsistence farmers raising mainly corn for their own family's consumption while women's primary responsibility was to run the house and the garden. Children's daily activities were primarily structured by their parents' work, which had priority and should not be interrupted by children (see Gaskins, 1999). There was little attention on how infants spent their time, with almost no adult interference, as long as they were safe. Mayan parents believe that young children develop and learn largely independently of caregivers' behavior toward them. Parents define their caregiver role in terms of ensuring the safety and well-being of the infant and in keeping the infant content by responding to its needs and desires, for example by soothing through frequent breastfeeding, but not in terms of stimulating interaction, modeling, or direct teaching (Gaskins, 2006).

Dutch families were chosen with the constraint that infants spent maximally 1 day per week in a day care, to ensure that home data collection was representative and comparable. Families had one to four children $(M=2.3)$.

In most Chinese families $(N=14)$, both parents worked and the grandparents took care of the child, which is a common pattern (Kun, 2007). Grandparents usually lived in the same apartment, forming a three-generation household. Four families employed a nanny for additional support. Single children in today's China are the center of attention, whose needs and interest are to be met above all else. This child-centered orientation has also been depicted in the media as the "little-emperorsyndrome." Children enjoy an elevated position at home, with multiple caregivers revolving around them (Goh \& Kuczynski, 2010).

\section{Design and Procedure}

Our study consisted of two independent parts: (a) scan sampling of daily activities and (b) video analyses of gestures. Each participating family was visited four times-twice for the scan sampling portion of the study, each lasting $2 \mathrm{hr}$ (totaling $4 \mathrm{hr}$ ), and twice for the video recordings, each lasting $30 \mathrm{~min}$ (totaling $1 \mathrm{hr}$ ). The four visits were timewise as contiguous as possible depending on the schedule of each family. The four visits represented random shots of daily life with the one constraint that infants had to be awake. If this was not the case, the observer postponed the meeting. For the Mayans, all four visits took place within 7 days, for the Dutch within 12 days, and for the Chinese within 8 days.

All data were collected by the same person (first author) who was not a member of either of the cultural groups. In all three groups a local assistant who spoke the native language was present for the introduction at the first appointment. Families were informed that we were interested in how infants spent their everyday life in different parts of the world. They were not informed about any of our hypotheses or specific dependent measures. Terms like social interaction, communication, or gestures were not mentioned. Families were asked to continue their everyday activities and simply ignore the observer who was sitting at an appropriate distance (e.g., in the corner of the room). Families were informed that they did not need to stay inside but could pursue their daily routines or whatever they would normally do when the observer was not around (including shopping, visiting friends, picking up older siblings from school, going to the playground, playing in the garden, etc.). In those cases the observer followed at an appropriate distance but close enough to continue observation.

For the scan sampling portion of the study, each family was observed once in the morning and once on a different day in the afternoon or early evening. The observer observed a focal infant online using a pen, coding sheet with predefined categories, and a stopwatch. The video recordings were done on another day after the scan sampling was completed, once in the morning and once in the afternoon or 
early evening. The infant and any interlocutor (including siblings, peers, other adults; not only biological parents) were video-recorded with a hand camera at an appropriate distance. For the Dutch and the Chinese sample a second camera recorded the scene on a tripod. This arrangement did not work out in the Mayan sample because families spent most of their time outside and walked around. Two families (one Mayan and one Dutch) preferred not to be video-recorded.

\section{Coding}

Scan sampling. The sessions were divided into 5-min observation units, totaling 48 observation units. Within each unit the occurrences of predefined activities were coded. Activities were conceptualized as "states" that extended in time (Bakeman \& Adamson, 1984). An activity had to last at least $30 \mathrm{~s}$. Brief actions or shorter periods of fluctuating activities were not coded. Activities were coded once per observation unit (see Table 1). Of main interest was the code "triadic joint action." Triadic joint action was coded when the infant and another person engaged together about an external entity or event, for example, by acting on an object ("joint engagement"; Bakeman \& Adamson, 1984). Other categories coded for "primary care" (attending to infants' biological needs), "social orientation" (observing other people), and "individual activities" (infants acting on their own; being self-absorbed). To account for the inverse relation between number and duration of activities in a unit, we divided a given activity in a unit by the sum of all other activities in that unit. We analyzed the mean proportion of time spent in each of the five activity categories across the 5-min units.

We also coded for the distance to the nearest potential interlocutor to check whether the availability and thus possibility to communicate was different across the three cultures. Distance coding was independent of the activity coding but followed the same rules. Five distances fell into three main categories: (a) attached: infants are attached to a caregiver's body, or infant and caregiver touch each other intentionally; (b) around: infant and caregiver are within reach or in view; and (c) away: caregivers are out of infants' view.

Reliability of the observer could only be assessed in retrospect by having the observer and a second assistant independently watch six of the video recordings (two from each culture, totaling 36 observation units) and use the same online scan sampling procedure. Agreements for the activities

Table 1

Coding Categories for Scan Sampling

\begin{tabular}{|c|c|c|c|}
\hline Categories & Activities & Definition & Examples \\
\hline \multirow[t]{2}{*}{ Interaction } & Triadic joint action & $\begin{array}{l}\text { Infant and interlocutor are } \\
\text { engaged about an external } \\
\text { entity or event }\end{array}$ & $\begin{array}{l}\text { Interlocutor and infant play with toys } \\
\text { Interlocutor and infant look at pictures/books }\end{array}$ \\
\hline & Dyadic interaction & $\begin{array}{l}\text { Infant and interlocutor interact } \\
\text { with each other without an } \\
\text { object }\end{array}$ & $\begin{array}{l}\text { Interlocutor makes funny faces for the infant } \\
\text { Interlocutor twists infant around in the air; } \\
\text { holds infant on her hands and walks around } \\
\text { with her }\end{array}$ \\
\hline \multirow[t]{2}{*}{ Primary care } & Washing/dressing & Infant is washed/dressed & $\begin{array}{l}\text { Person washes/bathes/dresses infant } \\
\text { Person changes infants' diaper }\end{array}$ \\
\hline & Feeding & Infant is fed & $\begin{array}{l}\text { Mother breastfeeds infant } \\
\text { Person feeds infant with crackers, tortillas, etc. }\end{array}$ \\
\hline $\begin{array}{l}\text { Social } \\
\text { orientation }\end{array}$ & Watching other people & $\begin{array}{l}\text { Infant watches other people } \\
\text { (without being involved in the } \\
\text { event herself) }\end{array}$ & $\begin{array}{l}\text { Infant watches mother talking to the shop } \\
\text { assistant } \\
\text { Infant watches other children running around }\end{array}$ \\
\hline \multirow[t]{4}{*}{$\begin{array}{l}\text { Individual } \\
\text { activities }\end{array}$} & Object play & Infant plays with an object by herself & $\begin{array}{l}\text { Infant plays with a toy } \\
\text { Infant picks leaves from a bush }\end{array}$ \\
\hline & Moving & Infant moves around by herself & $\begin{array}{l}\text { Infant crawls on the floor } \\
\text { Infant walks around in a walker }\end{array}$ \\
\hline & Eating & Infant eats by herself & $\begin{array}{l}\text { Infant drinks from her bottle } \\
\text { Infant eats apple pieces }\end{array}$ \\
\hline & $\begin{array}{l}\text { Unengaged } \\
\text { Other }\end{array}$ & $\begin{array}{l}\text { Infant does nothing (not even watches)- } \\
\text { just sits, lies around being self-absorbed }\end{array}$ & $\begin{array}{l}\text { Infant lies in a hammock doing nothing } \\
\text { Infant sits on caregiver's lap doing nothing }\end{array}$ \\
\hline
\end{tabular}


and the distance coding were excellent, respectively Cohen's Kappas $=.947 \quad(N=360) \quad$ and $\quad .924$ $(N=180)$.

Video analyses of gestures. We coded deictic gestures produced by and for the infant. As with triadic interactions, deictic gestures involve a referential triangle of sender, recipient, and object or event. They serve the primary function of directing attention to the object or event. POINT: The arm is either fully or half extended toward an object or event (close or distant). We distinguished indexfinger points (the index finger is extended relative to other fingers) from whole-hand points (no indexfinger extension). SHOW: Hand holds out an object, arm is extended toward a person. PLACE: Object is placed in front of a person, within her personal space, to direct attention to it (see also Clark, 2003). Mere object transfer was not counted. OFFER: Hand holds an object that is brought close to a person, so she can take it. Interlocutors' gestures had to be directed ostensively at the infant as a recipient-gestures to third parties were not counted as direct input.

Two research assistants, naive to the purpose of our hypotheses, coded the videos with the computer-based program ELAN. They coded equal portions of the three cultural samples and an overlap of 9 infants (20\%; 3 infants per culture) to establish reliability. The assistants' coding of infants' and others' gestures was highly correlated and statistically not different from each other (for infants, all $r_{\mathrm{s}}>.948, p \mathrm{~s}<.001, t \mathrm{~s}<1$; for others, all $r_{\mathrm{s}}>.913$, ps $\leq .001$, ts $<1$ ). Since data revealed great variability within cultures on several measures and diverged significantly from the normal distribution (Kolmogorov-Smirnov tests, $p \mathrm{~s}<.05$ ), data were subjected to square root transformations (Osborne, 2002). For ease of interpretation, the figures display uncorrected means.

\section{Results}

\section{Scan Sampling}

Triadic joint action. A 3 (culture) $\times 2$ (age group) analysis of variance (ANOVA) confirmed our prediction. Figure 1 shows that Mayan infants spent less time in triadic interactions compared to Dutch infants, who in turn spent less time compared to Chinese infants: linear contrast, $F(2,42)=46.44$, $p<.0005$; planned comparisons (LSD) based on the overall variance of the ANOVA, respectively, $p=$ $.035 ; p<.0005$. There was a main effect of age with more triadic interaction in the older age group, $F(2$,

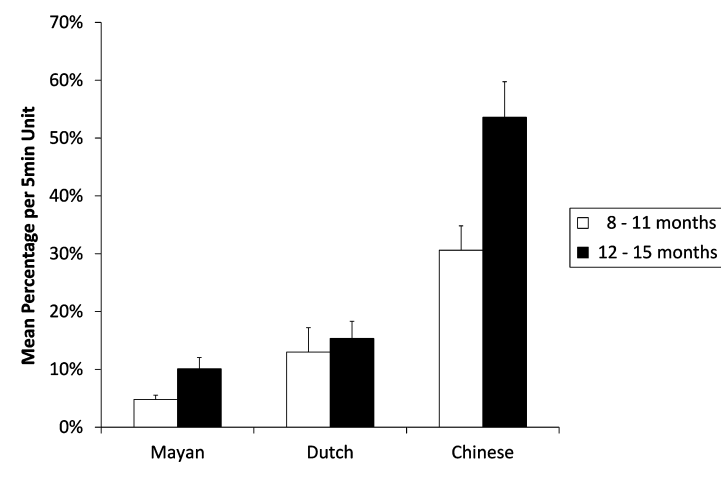

Figure 1. Triadic joint action between infant and interlocutor.

42) $=11.10, p=.002$, but this effect did not interact with culture.

Distance. A 3 (distance) $\times 3$ (culture) ANOVA revealed a significant main effect of distance, $F(2$, $90)=113.39, p<.001$, which interacted with culture, $F(4,90)=10.02, p<.001$. Simple effects (univariate) revealed that cultures varied significantly from one another in all three distances, all $F_{s}(2,45)>5.9$, $p s<.005$. Pairwise comparisons (LSD) based on the overall variance of the ANOVA showed that Dutch infants were less often attached compared to Mayan and Chinese infants (both $p s<.0005$ ), and instead more often around (respectively, $p=.002 ; p=.019$ ) or away (both $p$ s <.0005); see Figure 2. Mayan and Chinese infants did not differ significantly from one another on either of the distances.

Other activities. Figure 3 shows the mean proportion of all activities for each of the three cultures. In all three cultures infants spent about equal time with their caregivers in primary care. A significant one-way ANOVA, $F(2,45)=18.0, p<.0005$, revealed that Chinese infants spent less time in individual activities than Mayan and Dutch infants, presumably reflecting Chinese caregivers' role in creating triadic play episodes (post hoc comparisons [LSD] based on the overall variance, both

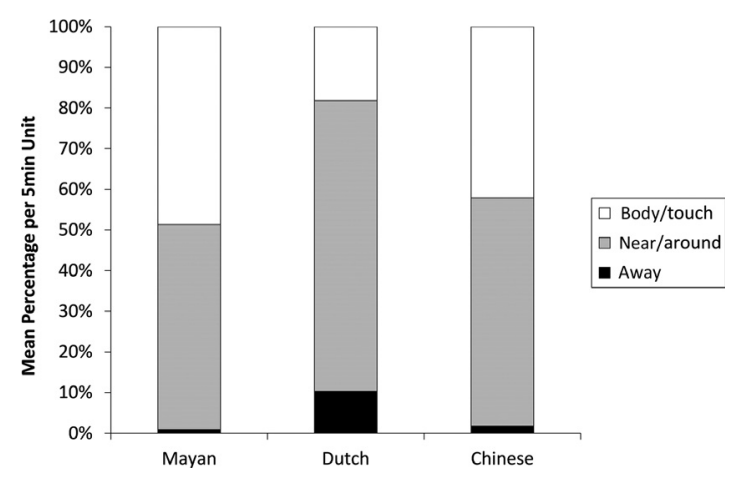

Figure 2. Interlocutor distance. 


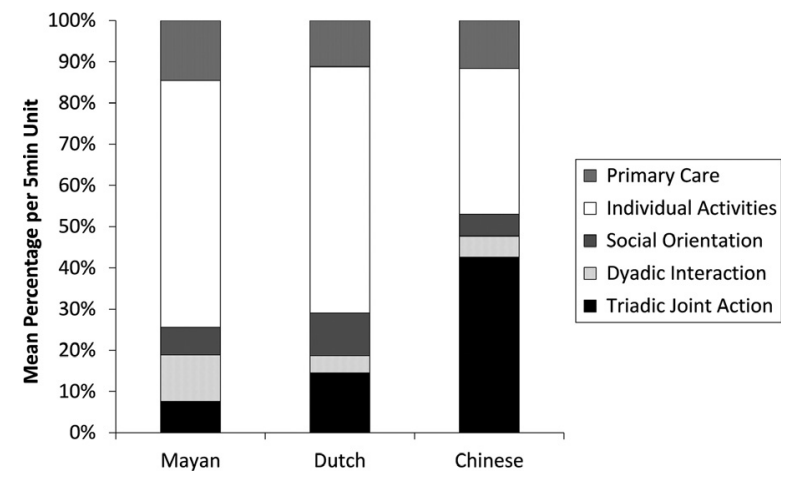

Figure 3. Daily activities.

ps $<.0005)$. A further significant one-way ANOVA, $F(2,45)=5.59, p<.007$, revealed that Mayan infants spent more time in dyadic interaction with their caregiver than Dutch and Chinese dyads (post hoc comparisons [LSD] based on the overall variance respectively, $p=.028, p=.002)$. Finally, a significant one-way ANOVA, $F(2,45)=4.4, p<.018$, revealed that Dutch infants spent slightly more time watching other people than Mayan or Chinese infants (post hoc comparisons [LSD] based on the overall variance respectively, $p=.041 ; p=.006$ ).

\section{Video Analyses of Gestures}

Interlocutors. A 3 (culture) $\times 2$ (age group) ANOVA confirmed our prediction. The upper left panel of Figure 4 shows that Mayan interlocutors gestured less compared to Dutch interlocutors, who in turn gestured less compared to Chinese interloc-
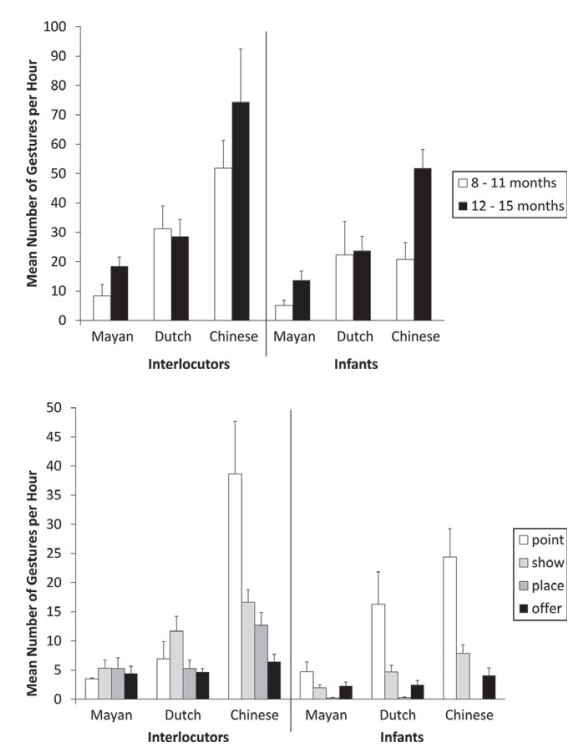

Figure 4. Upper panel: interlocutors' and infants' gestures. Lower panel: gesture types used by interlocutors and infants. utors: linear contrast, $F(2,40)=15.94, p<.0005$; planned comparisons [LSD] based on the overall variance of the ANOVA, respectively, $p=.050$; $p<.0005$. There was a main effect of age with more gestures to infants in the older age group, $F(1,40)=5.14, p=.029$. Age and culture interacted only marginally, $F(2,40)=2.89, p=.069$. Visual inspection of the data suggests an absence of age differences in the Dutch but not in the Mayan and Chinese samples.

The lower left panel of Figure 4 shows the types of interlocutors' gestures directed to infants across cultures. A 4 (gesture) $\times 3$ (culture) ANOVA revealed main effects of gesture, $F(3,129)=5.19, p=.002$, and culture, $F(2,43)=19.95, p<.001$, which were qualified by an interaction, $F(6,129)=6.49, p<.001$. Simple effects (univariate) revealed that cultures varied significantly from one another in all but the OFFER gesture, all $F \mathrm{~s}(2,43)>6.7, p \mathrm{~s}<.003$. For POINT, SHOW, and PLACE, pairwise comparisons (LSD) based on the overall variance revealed that Chinese interlocutors used POINT and PLACE significantly more often than Mayans and Dutch (all ps < .003). Chinese and Dutch used SHOW significantly more often than Mayans ( $p$ s $<.017)$, and Chinese tended to use SHOW more often than Dutch $(p=.090)$.

Within a culture, simple effects (multivariate) revealed that gesture types differed significantly from one another only in the Dutch and Chinese samples, respectively, $F(3,41)=4.3, p=.010$; $F(3,41)=15.94, p<.0005$. Pairwise comparisons (LSD) based on the variance of the overall ANOVA revealed that Chinese interlocutors used POINT more frequently than SHOW $(p=.011)$, SHOW and PLACE about equally often, and OFFER less than all other gestures $(p s<.009)$. Dutch interlocutors used SHOW more than all other gestures ( $p$ s < .013), with no significant differences between the other gestures. Mayan interlocutors used all gestures with equal frequency.

Infants. A 3 (culture) $\times 2$ (age group) ANOVA confirmed our prediction. As shown in the upper right panel of Figure 4, Mayan infants gestured less compared to Dutch infants, who in turn gestured less compared to Chinese infants: linear contrast, $F(2,40)=9.81, \quad p=.001$; planned comparisons (LSD) based on the overall variance of the ANOVA, respectively, $p=.045 ; p=.029$. There was a main effect of age with more gestures in the older age group, $F(1,40)=11.61, p=.002$, but this effect did not interact with culture.

The lower right panel of Figure 4 shows the types of infant gestures across cultures. A 4 
(gesture) $\times 3$ (culture) ANOVA revealed main effects of gesture, $F(3,129)=47.5, p<.001$, and culture, $F(2,43)=5.78, p=.006$, which were qualified by an interaction, $F(6,129)=4.98, p=.001$. Simple effects (univariate) revealed that cultures differed significantly from one another for POINT, $F(2,43)=7.01, \quad p=.002$, and SHOW, $F(2$, $43)=5.21, p=.009$, but not for OFFER and PLACE. Pairwise comparisons (LSD) based on the variance of the overall ANOVA confirmed for POINT the pattern of Mayan $<$ Dutch $<$ Chinese (respectively, $p=.030$, one-tailed; $p=.040$, one-tailed). For SHOW the pattern was similar, Mayan < Dutch $\leq$ Chinese (respectively, $p=.042$, one-tailed; $p=.081$, one-tailed).

Within a culture, simple effects (multivariate) revealed that gesture types differed significantly from one another in each of the three cultures, all three $F \mathrm{~s}(3,41)>5.14, p \mathrm{~s}<.004$. Pairwise comparisons (LSD) based on the variance of the overall ANOVA revealed that this was the case because PLACE was used in all three cultures significantly less compared to all other gestures (i.e., hardly or never; all $p s<.013)$. Chinese and Dutch infants used POINT more often than SHOW (respectively, $p<.0005 ; p=.030$ ), and SHOW more often than OFFER (respectively, $p=.003 ; p=.036$ ). In contrast, Mayan infants used these gestures with equal frequency.

Figure 5 shows that only 4 of the Mayan infants $(27 \%)$ pointed with the index finger (with a mean number of points $=6.8$ ). This was in contrast to 11 Dutch infants $(72 \%$; mean number of points $=14.5)$ and 14 Chinese infants $(88 \%$; mean number of points $=17.0$ ). The number of index-finger pointers was significantly different across the three groups, $\chi^{2}(2,46)=13.3, p=.001$. Importantly, the 4 Mayan infants who pointed with the index finger were in

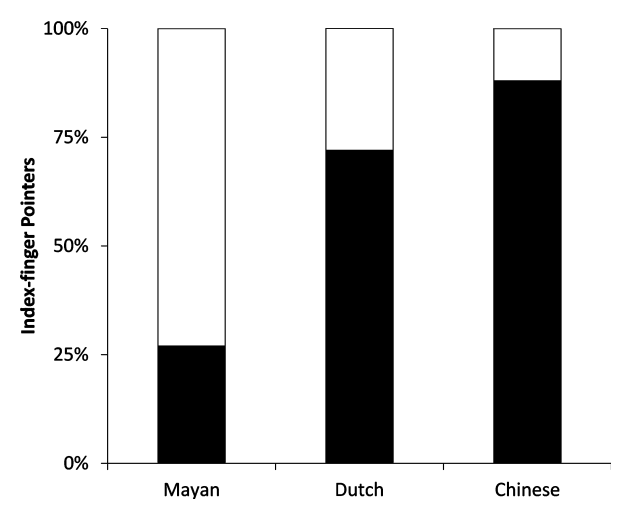

Figure 5. Percentage of index-finger pointers. the older age group above 12 months of age. Conversely, the 4 Dutch and 2 Chinese infants who did not yet point with the index finger were in the younger age group and all at or below 10 months of age.

\section{Predictors of Infant Gestures}

In a final analysis we tested whether differences in infants' gestures could be determined by positive correlations with interlocutors' gestures and triadic social interactions, even when controlling for culture. There were substantial correlations between interlocutors' gestures and infants' gestures, $r(d f=$ $46)=.626, p<.0005$, and between triadic social interactions and infants' gestures, $r(d f=46)=.668$, $p<.0005$. Positive partial correlations remained when controlling for culture (dummy-coded), respectively, $r($ par,$d f=42)=.414, p=.005 ; r($ par,$d f=42)=.480$, $p=.001$, and when controlling for both culture and age (continuous), $r($ par, $d f=41)=.273, p=.038$, one-tailed; $r($ par, $d f=41)=.251, p=.052$, one-tailed.

In addition, partial correlations revealed that interlocutors' gestures and triadic social interactions were interrelated, $r($ par, $d f=41)=.405, p=.007$. We investigated whether this interrelation differentially affected the correlations with infants' gestures. Infants' gestures did not correlate significantly with interlocutors' gestures when controlling for triadic social interactions, $r($ par, $d f=43)=.215, p=.165$. However, infants' gestures remained correlated with triadic social interactions when controlling for interlocutors' gestures, $r($ par, $d f=43)=.364$, $p=.014$. These findings thus suggest a more direct link between triadic social interaction and infants' gestures.

\section{Discussion}

On- and off-line observations of infants' engagement in joint actions and gestural communication revealed commonalities and differences across very different cultural settings. Despite vast sample differences, for example, in societal structure, parental beliefs, economy, educational and socialization goals, geographical and physical layout, daily structure and family size, we observed in all cultural settings that infants were engaged in triadic bouts of joint action, that they were gestured for, and that they gestured themselves. All four types of deictic gestures were present in the very different cultural settings. These findings support the assumption that joint action and deictic gestures are universally 
existing prelinguistic behaviors, and corroborate recent findings of a prelinguistic gestural universal of human communication in the specific case of index-finger pointing (Liszkowski et al., 2012).

The current study asked whether differences in the extent to which infants are immersed in social interaction and gestural communication would impact infants' use of gestures. Results revealed systematic differences between sociocultural settings in the frequency with which interlocutors engaged infants in joint actions and gestural communication. Our method of natural observations captured these real-life differences better than a previous study (Liszkowski et al., 2012) which used a prestructured elicitation format and probed only for the existence of a behavior within a given context, without controlling for its natural occurrence or cultural relevance.

The cultural group differences in the social-interactional "input" to infants did not simply result from interlocutors' proximity. Although Yucatec Mayans and Shanghai Chinese were on the opposite extremes of our social interaction measures, they did not differ in caregiver proximity: Infants were equally often at or around caregivers and available for interaction. Thus, we can exclude the possibility that proximity selectively prevented (or enhanced) joint actions and gestures. The differences also did not result from differences in mobility constraints. Mayan infants were not constrained to interact by being swaddled or carried in tight shawls. Instead, they could freely move most of the time. The number of potentially available interlocutors also cannot account for the differences we observed. For example, Mayan infants usually encountered more people as they spent a lot of time outside compared to the Chinese infants who stayed often (but not exclusively) inside small flats. Also, Dutch infants were more often alone than infants in the other two samples, presumably due to the use of playpen. Further, like Chinese, most Dutch infants in the current study were firstborn, suggesting that birth order itself was not the only driving factor of the differences we observed. And although socioeconomic status (SES) and education have been associated with caretaking patterns (Richman, Miller, \& LeVine, 1992), our post hoc judgment is that the Shanghai parents did not seem to have a higher education or SES than the Dutch parents. Note also that the relative meaning of education and SES within a cultural setting may be difficult to compare across cultures. Importantly, the cultural samples were not different altogether, as we controlled for primary caretaking activities that were similar across settings. Thus, the current study revealed systematic quantitative differences in the amount of triadic interactions and deictic gestures to which young infants are exposed. These differences appeared on the level of broadly defined cultural settings (Whiting, 1980), which constitute the developmental and ecocultural niches within which infants develop (see Super \& Harkness, 1986; Weisner, 2002).

Having established quantitative group differences in the interactional input, this allowed us to test the hypothesis that social-interactional input influences infants' prelinguistic gestures from early on. The natural input differences to age-matched infants of different cultural groups provided quasiexperimental independent conditions for a direct test. This is a lucky situation as the experimental suppression of joint acting and communicating with infants within a cultural setting is difficult, if not impossible, for ethical reasons. As predicted by the hypothesis, infants' prelinguistic gestures differed across cultural settings in the same way as their social-interactional input. Dutch infants who were exposed to more interactional input than Mayan infants gestured more, yet Chinese infants who were exposed to more interactional input than Dutch infants gestured still more. The specific pattern across three cultural samples makes it unlikely that any specific characteristic of one cultural sample alone accounted for these differences. Further, all infants were age-matched across the three cultural samples, suggesting that cultural differences impact infants' gesture use from early on.

The analysis of index-finger pointing supports the interpretation on the individual level. Index-finger pointing has previously been shown to be a milestone in communicative and social-cognitive development (Liszkowski \& Tomasello, 2011; Rowe \& Goldin-Meadow, 2009). In the current study, index-finger pointing was more frequent than other gestures and accounted for most of the group differences in infants' gestures. On an individual level, while all infants gestured at least once (except for two of the youngest infants in each of the Dutch and the Yucatec Mayan samples), index-finger pointing had emerged in only a few, older Mayan infants, while it had already emerged in the majority of the Dutch, and almost all Chinese infants. Research has revealed a correlated usage of parent and infant pointing across various cultural settings (Liszkowski \& Tomasello, 2011; Liszkowski et al., 2012), and this relation presumably mediates later word acquisition and preschooler's vocabulary size (Rowe \& Goldin-Meadow, 2009). The current find- 
ing on the individual level suggests that the very emergence of this developmental milestone is itself mediated by the amount of earlier social-interactional experience.

Our correlation analyses further revealed that the relation between social-interactional input and infants' gesture use was not mediated by culture, suggesting that social-interactional input is a universal developmental mechanism in the emergence of prelinguistic communication. When looking at the contributions of joint action and deictic gestures separately, the set of partial correlations revealed that infants' gestures were not predicted by interlocutors' gestures once we controlled for the time spent in joint action. The analyses on the frequency of the different gesture types further suggest that there was no direct one-to-one correspondence in the forms of interlocutors and infants' gestures. For example, as displayed in Figure 4, Dutch infants were exposed to more SHOW gestures but used more POINT than SHOW gestures; and although infants were exposed to the PLACE gesture they hardly ever used it. Presumably, deictic gestures do not emerge simply by mimicry alone. Instead, infants' gesture use may in part be mediated by a form-independent use of reference in the sense that acting on objects brings these to the attention of others. Indeed, infants' gestures were predicted by the time spent in joint action, and the relation remained when controlling for the amount of gestures directed at them. Word learning studies have shown that time spent in joint engagement is predictive of vocabulary size (Tomasello \& Farrar, 1986). Based on the current findings it is likely that not only verbal reference, but even the very concept of prelinguistic reference itself emerges within shared activities, in which others' actions structure infants' attention to objects.

An alternative possibility would have been that socialization processes become effective only after infants participate actively in social exchanges. In this case one would have expected no cultural differences early in infancy. This alternative was not supported. Yet, as a cross-cultural approach is only quasi-experimental in that there is never full control over the independent variable, it is at least theoretically possible that a nonsocial factor (e.g., one under genetic control) could account for the pattern of group differences. However, to date it is unknown whether there is relevant genetic variation in the expression of infant gestures, and we would find it unlikely that this variation would pattern across the three cultures in just the same way our behavioral findings did. A Gene $\times$ Environment interaction, as for example in the case of linguistic tone (Dediu, 2011), remains of course a possible, albeit to date speculative, interpretation of the current findings. Note also that while nutritional deficiencies may have an effect on early development (see Gorman, 1995), there was no known history of malnutrition in our samples, and certainly not in a way plausibly explaining the obtained differences between all three cultures. Instead, the current findings are consistent with the interpretation that the interactional input influences the very emergence of infants' prelinguistic gestures.

It is also unlikely that the $1 \mathrm{hr}$ of video recording was too short to capture instances of index-finger pointing in some samples. First, the observation interval was equal in all three cultures. Second, the observation interval was long enough to yield ample use of other object-directed gestures. Third, infants who did point with the index finger clearly pointed more than once per hour (also in the Mayan sample). It is also implausible that differences in the amount of items to point at, especially toys, drove the differences. First, the Yucatec Mayan infants who did not point used other, object-directed gestures, which must mean that there were objects to gesture with. Second, pointing increased with age, while the environment remained the same, again showing that there were items to point at, once the behavior had emerged.

The current study did not distinguish between the finer grained, mostly conceptual distinction of "active" and "passive" joint engagement, that is, respectively, how many of the joint bouts were initiated and maintained by the infant, and how many were scaffolded by the interlocutor. However, Bakeman and Adamson (1984) found that most of the shared activities in that age range are still scaffolded by caregivers, which also corresponds to our anecdotal observations. Another aspect that might warrant further investigation is whether joint actions differ also qualitatively across cultures, and whether certain types of joint activity will be more-or less-inductive to infant gesturing (see Puccini, Hassemer, Salomo, \& Liszkowski, 2010). Note also that we did not obtain a clear indication of "optimal" input: While Dutch infants, taken as a reference sample to most developmental literature, received more input than Yucatec Mayan infants, they received much less input than the Shanghai Chinese infants.

Explaining the origins of interlocutors' group differences touches upon the deeper, historical origins of cultural differences. Perhaps it is less likely that there is a direct link to the independent-interdependent socialization goals dichotomy that is often invoked in cross-cultural research (e.g., Keller, 2007), 
as both Yucatec Mayans and Shanghai Chinese have characteristics of following interdependent socialization goals (albeit in rural vs. urban settings; cf. Kagitcibasi, 2005). More directly, culturally transmitted beliefs about infants and development, environmental factors and habitual practices that frame the business of daily life, education, as well as available cultural tools, presumably all affect the style and frequency of social interaction with nonverbal infants (e.g., Chisholm, 1981; Harkness \& Super, 1996; Richman et al., 1992).

Development takes place within sociocultural activity. To benefit from and participate in sociocultural activities, one needs some fundamental communication skills. The current study reveals that the emergence of prelinguistic gestures is already mediated by social-interactional experience. The analyses of natural input differences across different sociocultural settings provided quasi-experimental evidence for a socially mediated emergence of prelinguistic deictic gestures in infancy. Our findings thus support sociocultural accounts of development already before language or other symbolic tools are in play. It is a reasonable assumption that others' actions structure the ontogeny of human communication and social cognition from early on.

\section{References}

Bakeman, R., \& Adamson, L. B. (1984). Coordinating attention to people and objects in mother-infant and peerinfant interaction. Child Development, 55, 1278-1289.

Brown, P. (2000). Conversational structure and language acquisition: The role of repetition in Tzeltal. Journal of Linguistic Anthropology, 8, 197-221.

Carpendale, J. I. M., \& Carpendale, A. B. (2010). The development of pointing: From personal directedness to interpersonal direction. Human Development, 53, 110-126.

Carpendale, J., \& Lewis, C. (2004). Constructing an understanding of mind: The development of children's social understanding within social interaction. Behavioral and Brain Sciences, 27, 79-96.

Carpenter, M., Nagell, K., \& Tomasello, M. (1998). Social cognition, joint attention, and communicative competence from 9 to 15 months of age. Monographs of the Society for Research in Child Development, 63(4, Serial No. 255).

Chisholm, J. S. (1981). Residence patterns and the environment of mother-infant interactions among the Navajo. In T. Field (Ed.), Culture and early interactions (pp. 3-19). Hillsdale, NJ: Erlbaum.

Clark, H. H. (2003). Pointing and placing. In S. Kita (Ed.), Pointing: Where language, culture, and cognition meet (pp. 243-268). Mahwah, NJ: Erlbaum
Dediu, D. (2011). A Bayesian phylogenetic approach to estimating the stability of linguistic features and the genetic biasing of tone. Proceedings of the Royal Society of London B, 278, 474-479.

Feiring, C., \& Lewis, M. (1981). Middle class differences in the mother-child interaction and the child's cognitive development. In T. Field (Ed.), Culture and early interactions (pp. 63-90). Hillsdale, NJ: Erlbaum

Fong, V. L. (2007). Parent-child communication problems and the perceived inadequacies of Chinese only children. Ethos, 35, 85-127.

Gaskins, S. (1996). How Mayan parental theories come into play. In S. Harkness \& C. M. Super (Eds.), Parents' cultural belief systems: Their origins, expressions, and consequences (pp. 345-363). New York: Guilford

Gaskins, S. (1999). Children's daily lives in a Mayan village: A case study of culturally constructed roles and activities. In A. Göncü (Ed.), Children's engagement in the world (pp. 25-81). Cambridge, UK: Cambridge University Press.

Gaskins, S. (2000). Children's daily activities in a Mayan village: A culturally grounded description. Journal of Cross-Cultural Research, 34, 375-389.

Gaskins, S. (2006). Cultural perspectives on infant-caregiver interaction. In N. J. Enfield \& S. C. Levinson (Eds.), Roots of human sociality (pp. 279-298). Oxford, UK: Berg.

Goh, E. C. L. (2006). Raising the precious single child in urban China: An intergenerational joint mission between parents and grandparents. Journal of International Relationships, 4, 7-28.

Goh, E. C. L., \& Kuczynski, L. (2010). Agency and power of single children in multi-generational families in urban Xiamen, China. Culture E Psychology, 15, 506-532.

Goldin-Meadow, S., \& Saltzman, J. (2000). The cultural bounds of maternal accommodation: How Chinese and American mothers communicate with deaf and hearing children. Psychological Science, 11, 307-314.

Gorman, K. S. (1995). Malnutrition and cognitive development: Evidence from experimental/quasi-experimental studies among the mild-to-moderately malnourished. Journal of Nutrition, 125, 2239S-2244S.

Harkness, S., \& Super, C. M. (Eds.). (1996). Parents' cultural belief systems: Their origins, expressions, and consequences. New York: Guilford.

Harkness, S., Super, C. M., \& van Tijen, N. (2000). Individualism and the "Western mind" reconsidered: American and Dutch parents' ethnotheories of the child. New Directions for Child and Adolescent Development, 87, 23-39.

Harris, M., Barlow-Brown, F., \& Chasin, J. (1995). The emergence of referential understanding: Pointing and the comprehension of object names. First Language, 15, 19-34.

Hewlett, B. S. (1996). Diverse contexts of human infancy. New York: Prentice Hall.

Ho, D. (1989). Continuity and variation in Chinese patterns of socialization. Journal of Marriage and Family, 51, 149-163. 
Iverson, J., \& Goldin-Meadow, S. (2005). Gestures paves the way for language development. Psychological Science, 16, 367-371.

Kagitcibasi, C. (2005). Autonomy and relatedness in cultural context. Journal of Cross-Cultural Psychology, 36, 403-422.

Keller, H. (2007). Cultures of infancy. Mahwah, NJ: Erlbaum.

Keller, H., Otto, H., Lamm, B., Yovsi, R., \& Kärtner, J. (2008). The timing of verbal/vocal communication between mothers and their infants: A longitudinal cross-cultural comparison. Infant Behavior $\mathcal{E}$ Development, 31, 217-226.

Kun, Z. (2007). 50\% plus of city's under-3s raised by grandparents. China Daily, 13 July, p. 5.

Lancy, F. (2010). Learning 'From nobody': The limited role of teaching in folk models of children's development. Childhood in the Past: An International Journal, 3, 79-106.

Lieven, E., \& Stoll, S. (2009). Language development. In M. Bornstein (Ed.), The handbook of cross-cultural developmental science (pp. 143-160). New York: Psychology Press.

Liszkowski, U. (2010). Deictic and other gestures in infancy. Acción Psicológica, 7, 21-33.

Liszkowski, U., Brown, P., Callaghan, T., Takada, A., \& De Vos, C. (2012). A prelinguistic gestural universal of human communication. Cognitive Science. 36, 698-713. doi:10.1111/j.1551-6709.2011.01228.x

Liszkowski, U., \& Tomasello, M. (2011). Individual differences in social, cognitive, and morphological aspects of infant pointing. Cognitive Development, 26, 16-29.

Osborne, J. (2002). Notes on the use of data transformation. Practical Assessment, Research E Evaluation, 8(6). Retrieved from http://PAREonline.net/getvn.asp? $\mathrm{v}=8 \& \mathrm{n}=6$

Puccini, D., Hassemer, M., Salomo, D., \& Liszkowski, U. (2010). The type of shared activity shapes caregiver and infant communication. Gesture, 10, 279-297.

Pye, C. (1986). Quiché Mayan speech to children. Journal of Child Language, 13, 85-100.
Pye, C. (1992). The acquisition of K'iche' Maya. In D. Slobin (Ed.), The cross-linguistic study of language acquisition, Vol. 3 (pp. 221-308). Hillsdale, NJ: Erlbaum.

Richman, A., Miller, P., \& LeVine, R. (1992). Cultural and educational variations in maternal responsiveness. Developmental Psychology, 28, 614-621.

Rowe, M. L., \& Goldin-Meadow, S. (2009). Differences in early gesture explain SES disparities in child vocabulary size at school entry. Science, 323, 951-953.

Schieffelin, B., \& Ochs, E. (Eds.) (1986). Language socialization across cultures. New York: Cambridge University Press.

Super, C. M., \& Harkness, S. (1986). The developmental niche: A conceptualization at the interface of child and culture. International Journal of Behavioral Development, 9, 545-569.

Tomasello, M. (2008). Origins of human communication. Cambridge, MA: MIT Press.

Tomasello, M., \& Farrar, M. J. (1986). Joint attention and early language. Child Development, 57, 1454-1463.

Tomasello, M., \& Todd, J. (1983). Joint attention and lexical acquisition style. First Language, 4, 197-212.

Vygotsky, L. S. (1978). Mind in society: The development of higher psychological processes. Cambridge, MA: Harvard University Press.

Weisner, T. S. (2002). Ecocultural understanding of children's developmental pathways. Human Development, 45, 275-281.

Weisner, T. S. (2009). Culture, development, and diversity: Expectable pluralism, conflict, and similarity. Ethos, 37, 181-196.

Whiting, B. (1980). Culture and social behavior: A model for the development of social behavior. Ethos, 8, 95-116.

Whiting, B., \& Whiting, J. (1975). Children of six cultures: A psycho-cultural analysis. Cambridge, MA: Harvard University Press. 